\title{
Seismic Response of Magnetically Levitated House
}

\author{
Yuji Miyamoto1,2, Takaharu Nakano², Atsushi Shimamura3 ${ }^{3}$, Kentaro Sho ${ }^{2}$ \\ ${ }^{1}$ Department of Architecture and Civil Engineering, Fukui University of Technology, Fukui, Japan \\ ${ }^{2}$ Graduate School of Engineering, Osaka University, Osaka, Japan \\ ${ }^{3}$ Development Department, Chemical Grouting Company, Tokyo, Japan \\ Email: miyamoto@arch.eng.osaka-u.ac.jp
}

How to cite this paper: Miyamoto, Y., Nakano, T., Shimamura, A. and Sho, K. (2022) Seismic Response of Magnetically Levitated House. Open Journal of Earthquake Research, 11, 1-17.

https://doi.org/10.4236/ojer.2022.111001

Received: December 27, 2021

Accepted: February 7, 2022

Published: February 10, 2022

Copyright $\odot 2022$ by author(s) and Scientific Research Publishing Inc. This work is licensed under the Creative Commons Attribution International License (CC BY 4.0).

http://creativecommons.org/licenses/by/4.0/ (c) (i) Open Access

\begin{abstract}
This study explored the seismic response of a house supporting base sides with a polymeric displacement control material and by magnetically levitating the foundation base. In this paper, we explore the possibility and efficacy of a seismic-isolated detached house as described above from both a shaking table experiment of model and three-dimensional finite element analysis. The seismic-isolated model showed stable response and its acceleration response was significantly reduced compared to the base-fixed model in the shaking table test. Three-dimensional finite element analysis was possible to simulate the experimental results. In the seismic response analysis of a full-scale detached house, the seismic-isolated model showed response reduction and its residual displacement was smaller than that of the sliding-base isolation model.
\end{abstract}

\section{Keywords}

Seismic Response Reduction, Magnetic Levitation, Shaking Table Test, 3D FEM

\section{Introduction}

The 1995 Hyogoken-Nanbu Earthquake caused extensive building damage. As a result, seismic isolation and response control buildings were rapidly developed in Japan as the main technological means for reducing the seismic response of buildings. The effectiveness of these seismic isolation and response control buildings in reducing damage has been demonstrated to a certain extent in the major earthquakes that have occurred since then. However, since the magnitude of ground motion predicted for a Nankai Trough mega-quake or for Tokyo inland 
earthquake exceeds the magnitude currently considered in designs, the limitations of seismic isolation and response control structures have been indicated [1]. Therefore, further advancement of response-reducing technology during earthquake is required.

The ground motion occurring at a site is input into a building under the influence of the dynamic interaction between the ground and the structure [2] [3]. The seismic response of a building can be significantly reduced if the foundation base is isolated from the ground and the transmission path of the ground motion is artificially excised. There are several methods for isolating the base of a foundation from the ground, such as using compressed air [4] [5] or the repulsive force of magnets [6] [7] [8] [9] [10] to levitate a structure. Using compressed air has been applied mainly to houses in recent years. In addition, Miyamoto, et al. [10] conducted model tests on a shaking table to confirm the seismic-response-reducing effects of a base-isolated building (we call a seismic-isolated structure). The building model is magnetically levitated using permanent magnets and the base sides are supported by soft, high damping artificial geomaterial to control horizontal displacement.

As an extension of existing research [10], this study explored the seismic response of a house supporting base sides with a polymeric displacement control material and by magnetically levitating the foundation base. Figure 1 shows the schematic configuration of the seismic isolated structure. The foundation base and the ground are isolated by the repulsive force of the magnet. Here, according to Earnshaw's theorem [11], the gravity acting on an object in the air cannot be stably supported by only the electromagnetic force from a static electric field and static magnetic field. Consequently, to stabilize a magnetically levitated house, it is necessary to control and periodically change the magnetic field or to support the building in a way that does not depend on electromagnetic force. Therefore, in this study, stable magnetic levitation was realized by supporting the sides of a foundation with soft and highly resilient displacement control materials. The purpose of this study is to obtain fundamental data to realize the seismic-isolated structure. In this paper, we explore the possibility and efficacy of a seismic-isolated detached house as described above from both a shaking table experiment of model and three-dimensional finite element analysis.

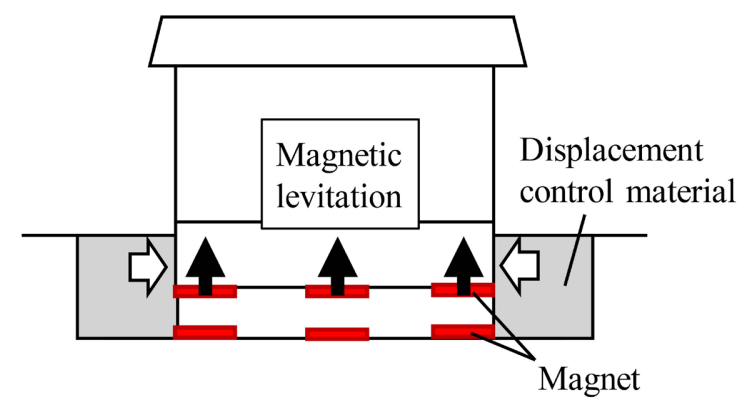

Figure 1. Diagram of seismic-isolated building with displace control by compound geo-material. 


\section{Shaking Table Experiment of Model}

\subsection{Experimental Conditions}

The experiment was carried out using a small shaking table (1.5 $\mathrm{m}$ in length and $0.7 \mathrm{~m}$ in width) owned by Osaka University. A cross-sectional view and photograph of the test body are shown in Figure 2 and Figure 3, respectively. The test body consists of a building model, magnet, and displacement control material in an acrylic container.

The building model consists of an aluminum foundation, steel columns, and a brass superstructure, and it represents a two-story detached house. The primary natural frequency of the building model when the base is fixed is confirmed to be $10.5 \mathrm{~Hz}$ according to free vibration tests. The total mass of the foundation and the superstructure is $13.9 \mathrm{~kg}$.

Four respective pairs of neodymium magnets are attached to the container and the building foundation base, and the foundation is isolated by their repulsive force. Figure 3 (b) shows that the building model levitates by magnetic force. The magnets are flat plates of $70 \mathrm{~mm} \times 70 \mathrm{~mm}$ in width and $5 \mathrm{~mm}$ in thickness. The residual magnetic flux density is approximately $1.2 \mathrm{~T}$ from the catalog value, and the distance between the upper and lower magnets is approximately $14 \mathrm{~mm}$ in actually measured values.

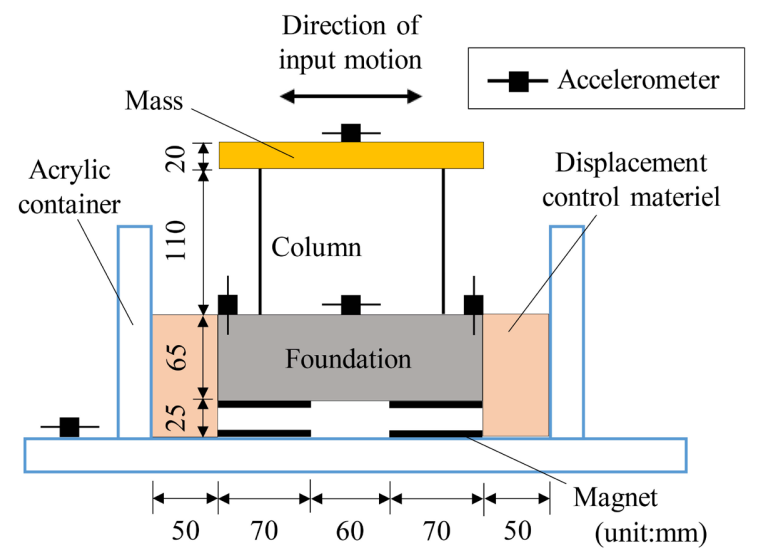

Figure 2. Section of specimen.

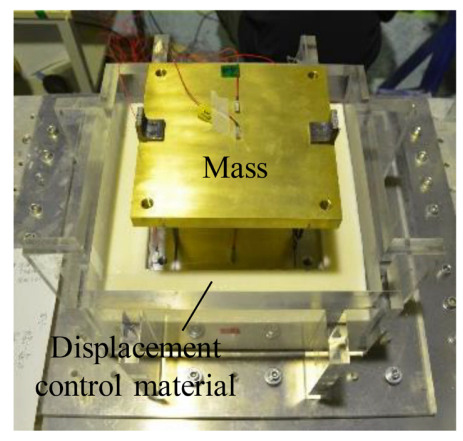

(a) Overview

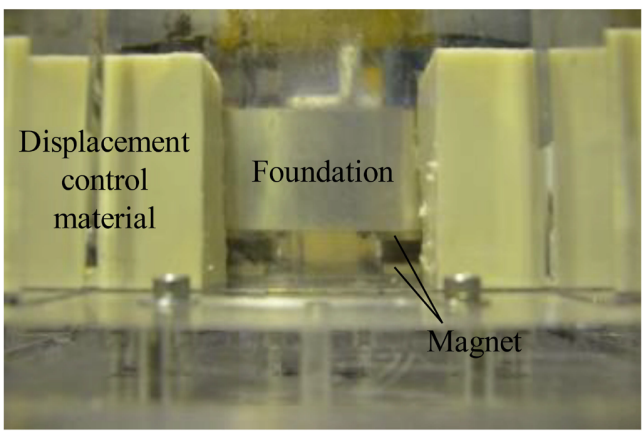

(b) Magnetic levitation of foundation

Figure 3. Specimen of shaking table test. 
Magnesium acrylate polymer is used as the material for the horizontal and vertical displacement control material supporting the base sides. This material is a polymer made by polymerizing an oxidizing and reducing agent after mixing with magnesium acrylate (monomer). The curing period required for polymerization reaction is approximately three days. Figure 4 shows the stress-strain relationship of the material according to a uniaxial compression test. It is shown that the material has high linearity even for large strains exceeding $10 \%$.

Figure 2 depicts the acceleration measurement setup. For the input earthquake ground motion, we standardized 1/5 times the time axis of the notification wave (random phase), which is the design ground motion that is equivalent to level 2 in Japan (RDM wave). Moreover, a wave observed on the surface by KiK-net Mashiki Station during the 2016 Kumamoto Earthquake (EW component) with the maximum velocity of $50 \mathrm{~cm} / \mathrm{s}$ and $1 / 10$ times the time axis (MSK wave), is used. The time axis of each input seismic wave was adjusted to match the dominant frequency of the seismic wave to the natural frequency of the building model. In the experiment, the amplitudes of these waveforms were increased in the order of $0.2,0.5,1.0$, and 1.5 times, and the RDM and MSK waves were input in the increasing order of the respective amplitudes. Figure 5 shows the acceleration time history waveforms of the RDM and MSK waves with 1.0 times the amplitude.

\subsection{Experiment Results}

Figure 6 shows the time waveforms of the horizontal acceleration measured at the top of the superstructure. For comparison within Figure 6, we superimposed the acceleration waveform in an experiment in which the base was fixed to a shaking table. For both the MSK and RDM waves, the response waveform of the

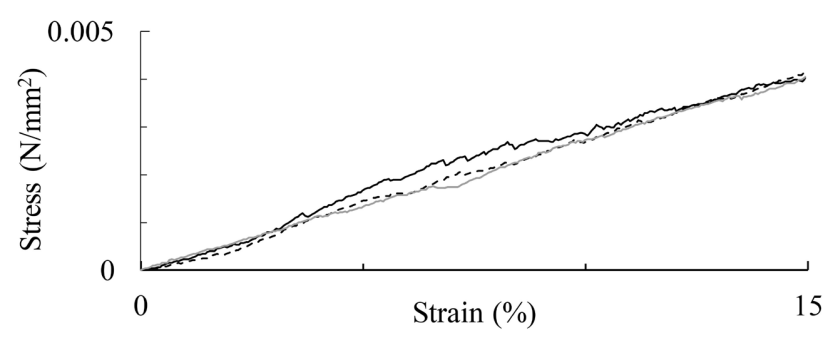

Figure 4. Result of uniaxial compression test for the material surrounding the base side.

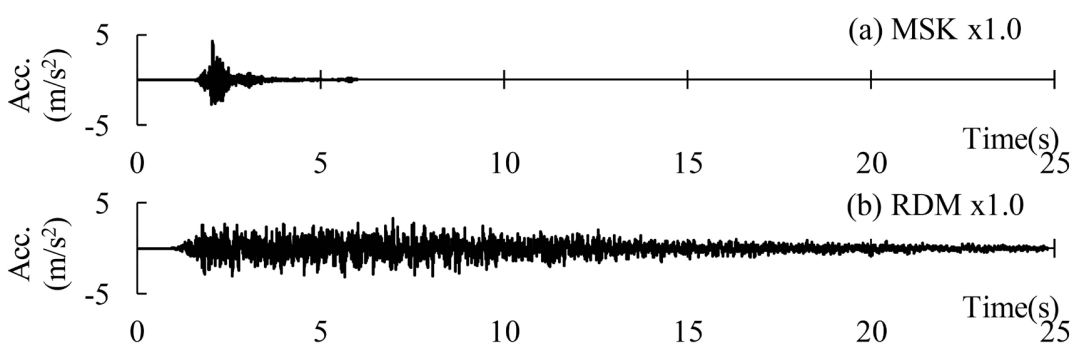

Figure 5. Acceleration history of input motion. 

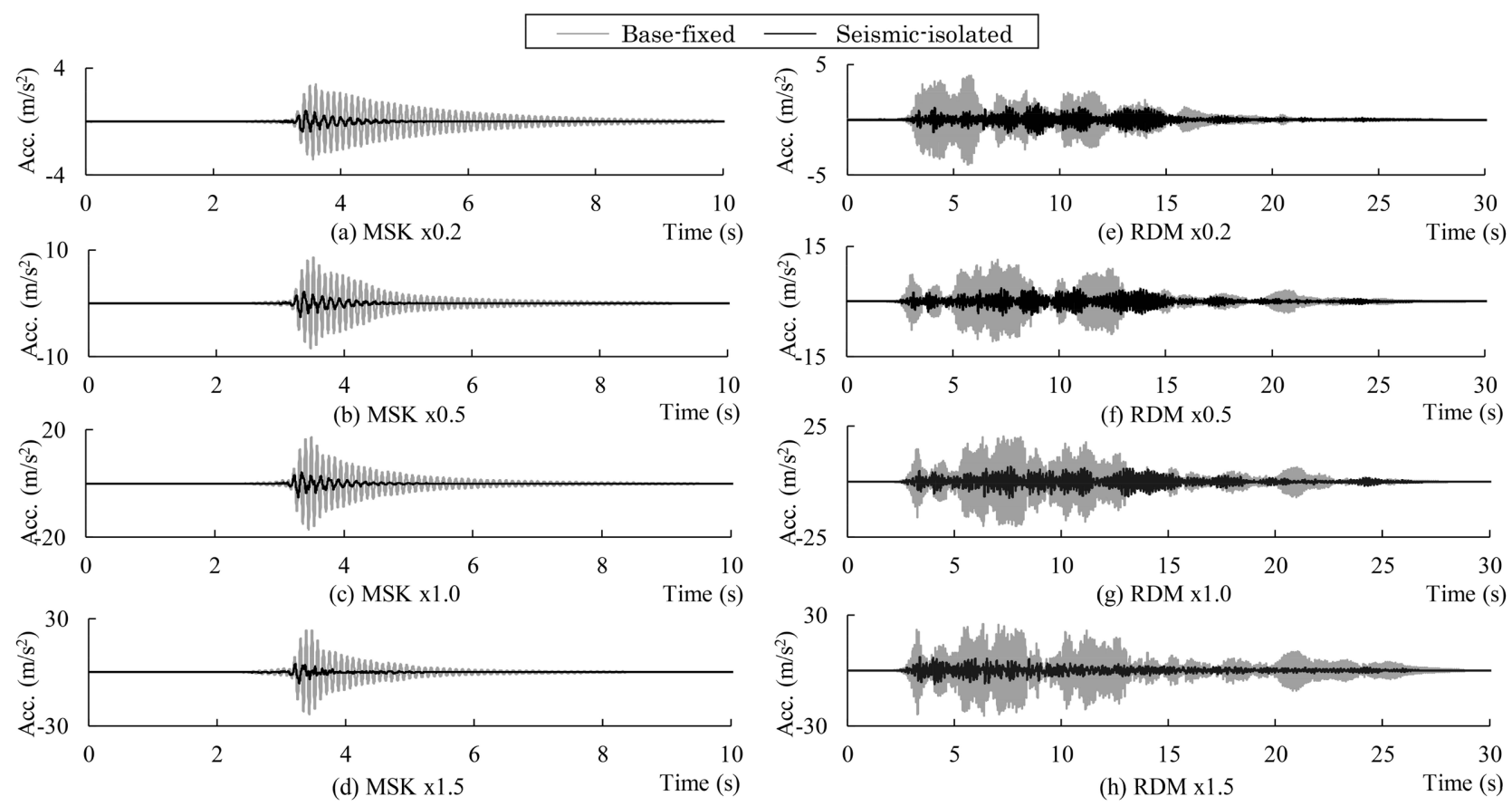

Figure 6. Acceleration history of mass.

superstructure of the seismic-isolated model of the experiment was remarkably reduced compared to the base-fixed model, regardless of the amplitude of the input waveform.

Figure 7 depicts a comparison of the Fourier acceleration amplification factor for the top of the superstructure in response to input between the seismic-isolated model and the base-fixed model. For both MSK and RDM waves, the dominant frequency of the seismic-isolated model is lower and the maximum amplification factor is smaller than that of the base-fixed model, regardless of input amplitude.

Figure 8 shows the Fourier acceleration amplification factor compared to the input amplitude for the top of the superstructure in response to input in the seismic-isolated model. Minimal change in dominant frequency and amplification factor due to input amplitude is observed for 1.0 times or less the MSK wave or 0.4 times or less the RDM wave. On the other hand, the dominant frequency is low and the maximum amplification factor is small for 1.5 times the input, confirming the nonlinearity of the test body response.

\section{Experimental Simulation by Three-Dimensional Finite Element Method}

\subsection{Modeling the Magnetic Force}

We explain the modeling of magnetic force for a simulation analysis model. The relationship between the resultant repulsive forces between magnets of a finite size and the distance between magnetic poles was calculated based on Coulomb's law. 


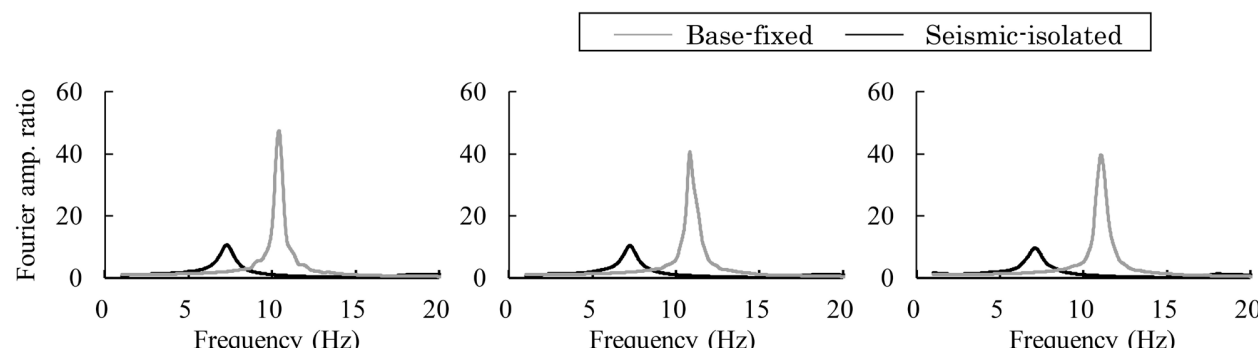

(a) MSK x0.2

(e) RDM $x 0.2$

(c) MSK x 1.0

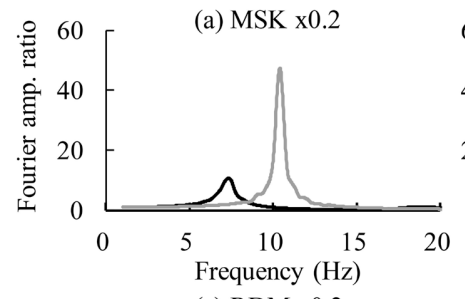

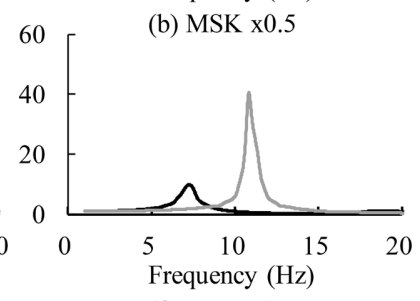

(f) $\mathrm{RDM} \times 0.5$

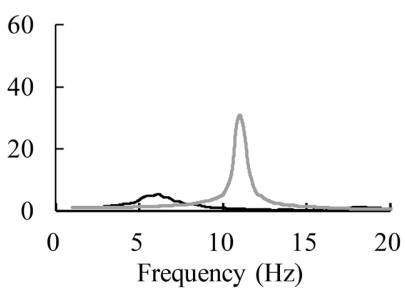

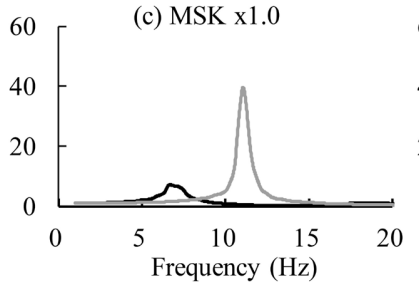

(g) RDM x 1.0

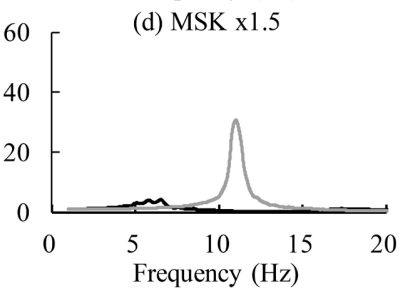

(h) RDM x 1.5
Figure 7. Fourier amplification factor of mass response to input motion.

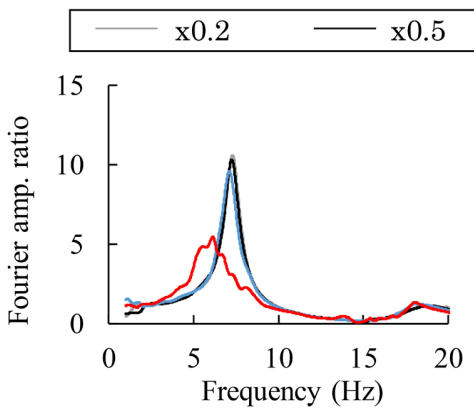

(a) MSK

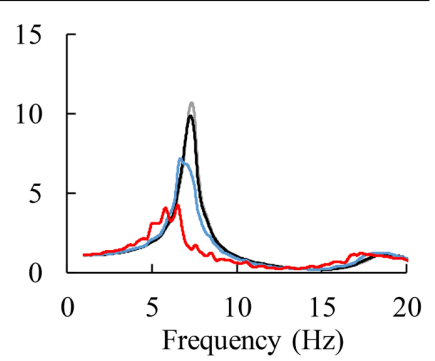

(b) RDM
Figure 8. Comparison of Fourier amplification factor of mass response to input motion between cases with different input levels.

\subsubsection{Repulsive Force between Monopoles}

A hypothetical particle without size, consisting only of a positive pole or a negative pole, is called a monopole. There are two monopoles, $\mathrm{A}$ and $\mathrm{B}$, with respective strengths $m$ and $m^{\prime}$, and respective position vectors $\boldsymbol{r}=(x, y, z)$ and $\boldsymbol{r}^{\prime}=\left(x^{\prime}, y^{\prime}, z-d\right)$. The $z$ component $F_{m z}$ of the repulsive force that A receives from $B$ is given by Equation (1) according to Coulomb's law.

$$
\begin{gathered}
F_{m z}=\left\langle\frac{m m^{\prime}}{4 \pi \mu} \frac{\boldsymbol{r}-\boldsymbol{r}^{\prime}}{\left|\boldsymbol{r}-\boldsymbol{r}^{\prime}\right|^{3}}, \boldsymbol{e}_{z}\right\rangle=\frac{m m^{\prime}}{4 \pi \mu} f(d) \\
f(d)=\frac{d}{\left\{\left(x-x^{\prime}\right)^{2}+\left(y-y^{\prime}\right)^{2}+d^{2}\right\}^{3 / 2}}
\end{gathered}
$$

Here, $\mu$ is the permeability of air, and we use the same value as the permeability of a vacuum $\left(=1.26 \times 10^{-6} \mathrm{~N} / \mathrm{A}^{2}\right)[12]$. In addition, $\boldsymbol{e}_{z}$ is a unit vector in the $z$ direction, and $\langle\boldsymbol{a}, \boldsymbol{b}\rangle$ represents the inner product of the vectors.

\subsubsection{Repulsive Force between Dipoles}

A dipole is defined as a pair of two monopoles having the same strength with 
opposite signs. Figure 9(a) shows two dipoles, A and B, with respective positive pole strengths, $m$ and $m$ '. The two dipoles are parallel to the $z$ axis with lengths $t$, and their positive poles face each other at distance $d$ in the $z$ direction. Here, the $z$ component $F_{d z}$ of the resultant repulsive force that dipole $\mathrm{A}$ receives from $\mathrm{B}$ is given by Equation (3).

$$
F_{\mathrm{d} z}=\frac{m m^{\prime}}{4 \pi \mu}\{f(d)+f(d+2 t)-2 f(d+t)\}
$$

\subsubsection{Repulsive Force between Magnets of Finite Size}

Figure 9(b) shows two magnets, $A_{1}$ and $A_{2}$, which are both orthogonal to the $\mathrm{z}$ axis, with their positive poles facing each other at distance $d$. The thickness of the magnets is $t$ and the absolute value of the residual magnetic flux density is $B_{r}$. Considering both magnets to be a collection of dipoles, as in Section 3.1.2, the $z$ component $\mathrm{d} F_{z}$ of the repulsive force that the micro-dipole $\mathrm{d} A_{1}$ of magnet $A_{1}$ receives from the micro-dipole $\mathrm{d} A_{2}$ of magnet $A_{2}$ is given by Equation (4).

$$
\mathrm{d} F_{z}=\frac{B_{r}^{2} \mathrm{~d} A_{1} \mathrm{~d} A_{2}}{4 \pi \mu}\{f(d)+f(d+2 t)-2 f(d+t)\}
$$

By integrating Equation (4), the $z$ component $F_{z}$ of the resultant repulsive force that magnet $A_{1}$ receives from $A_{2}$ is given by Equation (5).

$$
F_{z}=\int_{A_{2}} \int_{A_{1}} \mathrm{~d} F_{z}
$$

The planar shape of magnet $A_{1}$ is assumed to be rectangular, and the interval of integration is given by Equation (6).

$$
\left\{\begin{array}{l}
x_{1} \leq x \leq x_{2} \\
y_{1} \leq y \leq y_{2}
\end{array}\right.
$$

Thus, the integral with respect to $A_{1}$ in Equation (5) can be solved analytically, and Equation (7) is obtained.

$$
\begin{gathered}
F_{z}=\frac{B_{r}^{2}}{4 \pi \mu} \int_{A_{2}}\{h(d)+h(d+2 t)-2 h(d+t)\} \mathrm{d} A_{2} \\
h(d)=g\left(x_{2}, y_{2}, d\right)-g\left(x_{2}, y_{1}, d\right)-g\left(x_{1}, y_{2}, d\right)+g\left(x_{1}, y_{1}, d\right) \\
g(x, y, d)=\tan ^{-1}\left(\frac{\left(x-x^{\prime}\right)\left(y-y^{\prime}\right)}{d \sqrt{\left(x-x^{\prime}\right)^{2}+\left(y-y^{\prime}\right)^{2}+d^{2}}}\right)
\end{gathered}
$$

Here, $\tan ^{-1}()$ is the principal value of the arc tangent.

From Equation (7), the relationship between the distance between magnets $d$ and repulsive force $F_{z}$ can be obtained.

\subsubsection{Comparison with Experimental Results of Magnetic Force}

Figure 10 shows the repulsive force-magnetic distance relationship per one pair of magnets used in the shaking table experiment as a "theoretical curve". The "experimental values" in the figure are the distance measured between the magnets by placing a weight on top of the magnets. We find that the theoretical curve generally captures trends in experimental values. 


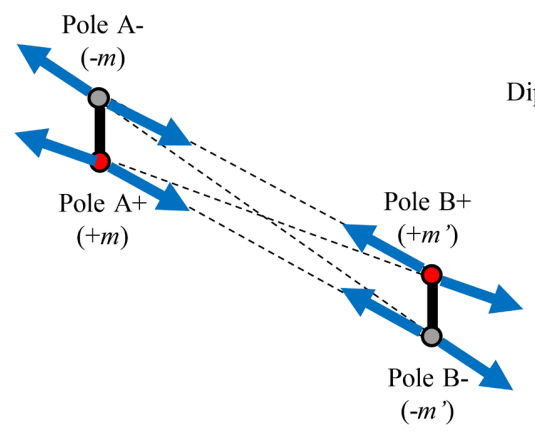

(a) Force acting on dipoles

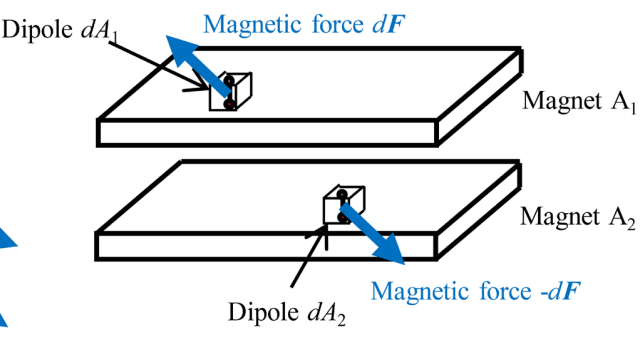

(b) Force acting on magnets

Figure 9. Model of magnet as group of dipoles.

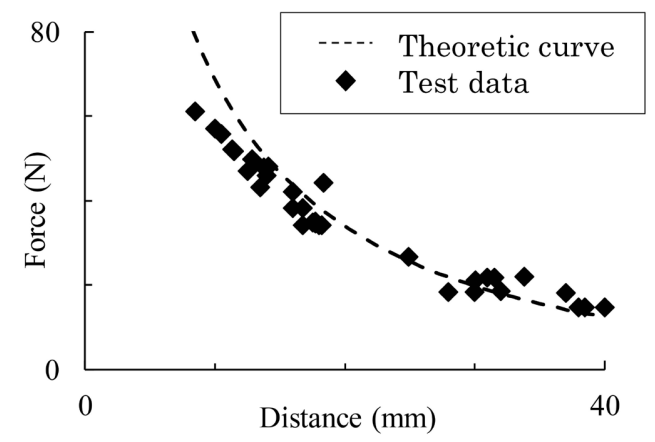

Figure 10. Relationship between magnetic force and distance of magnets.

\subsection{Simulation Analysis Model and Analysis Conditions}

Here, we perform a simulation analysis of the shaking table experiment described in Section 2 using the three-dimensional finite element method. The analysis code used is LS-DYNA. In addition, the subject of analysis will be the experiment inputting an MSK wave.

Figure 11 shows the analysis model. The base was modeled as a rigid solid element, and the superstructure is modeled as a linear mass system. The horizontal stiffness and the viscous damping were determined when the fixed-base primary natural frequency was $10.5 \mathrm{~Hz}$. The damping constant was $0.74 \%$, which matched the results of the excitation when the base was fixed. The material surrounding the base side was modeled with an elastic solid element. The Young's modulus of the material surrounding the base side was set to $0.0278 \mathrm{~N} / \mathrm{mm}^{2}$ based on the results of the uniaxial compression test, and Poisson's ratio was set to 0.4994 . Stiffness proportional damping was applied to the material surrounding the base side, and the damping constant at the primary natural frequency of the specimen was set to $14 \%$. Thus, the response of the top of the superstructure to the 0.2 times MSK wave input was consistent with the experiment results.

Since the repulsive force of a magnet varies with the distance between magnetic poles, the magnetic force behaves similar to a spring when the foundation is displaced vertically. Therefore, we modeled the vertical resistance of magnets as a linear axial spring. The vertical spring constant per magnet used in the 


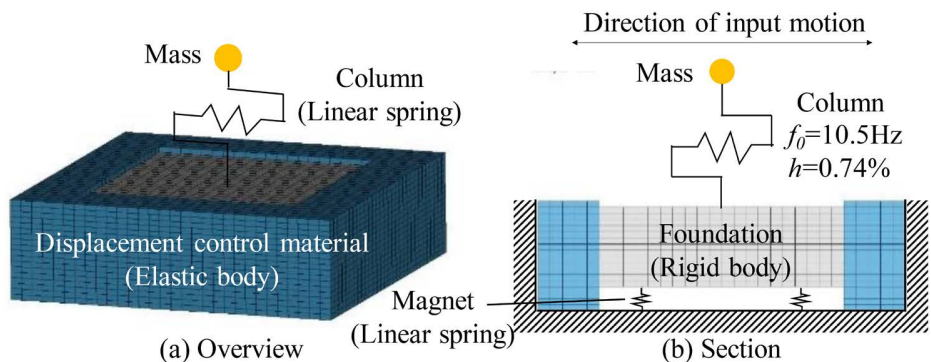

Figure 11. 3D FE model for shaking table test.

analysis was $3.48 \mathrm{~N} / \mathrm{mm}$, which is the slope of the tangent line of the theoretical curve shown in Figure 9, corresponding to the $14 \mathrm{~mm}$ distance between magnets in the experiment.

In the three-dimensional finite element model, the sides and bottom surfaces of the material surrounding the base side were assumed to be fixed conditions. In addition, analysis of two conditions (adhesion and debonding) was performed for the boundary surface between the base and the material surrounding the base side.

\subsection{Adhesion between Base and Material Surrounding the Base Side}

Figure 12 shows the acceleration time history waveform at the top of the superstructure. The analysis results for the 1.0 times or less input correspond well to the experimental results in terms of both amplitude and phase. The analysis result for the 1.5 times input is in good agreement with the experimental result up to approximately $3.3 \mathrm{~s}$; however, the amplitude was larger than the experimental results at later times.

\subsection{Debonding between Base and Material Surrounding the Base Side}

Here, we analyze the 1.5 times input considering the debonding between the base and the material surrounding the base side. In this analysis, we set the condition that the debonding first occurs when the tensile force acting on the contact surface reaches a certain tensile stress (hereafter, "tensile breaking stress"). The friction coefficient after debonding occurs is set to 0.4 .

Figure 13 shows the Fourier acceleration amplification factor at the top of the superstructure for the 1.5 times MSK wave input. Based on the results of an investigation varying tensile breaking stress $n$, the analysis results with $n=0.0036$ $\mathrm{N} / \mathrm{mm}^{2}$ correspond well to the experimental results. We thus adopt $n=0.0036$ $\mathrm{N} / \mathrm{mm}^{2}$.

Figure 14 shows the acceleration time history waveform at the top of the superstructure in comparing the analysis and experimental results. The results of the analysis with 1.0 times or less of input correspond well to the experiment results in terms of both amplitude and phase. We find that the analysis results with the 1.5 times input, which do not correspond well with the experimental results 
in the analysis-assuming adhesion between the base and the material surrounding the base side-correspond to the experimental results.

Figure 15 shows a deformation diagram of an analytical model considering debonding at the 1.5 times MSK wave input. However, the displacement is enlarged ten times in this figure. Some debonding is observed between the base and the material surrounding the base side. In addition, the material surrounding the base side not only resists the horizontal displacement of the base by expanding and contracting in the horizontal direction, but it also resists the rocking by expanding and contracting in the vertical direction. Thus, we find that the material surrounding the base side three-dimensionally resists the movement of the building model, which leads to stabilization of the seismic-isolated structure.

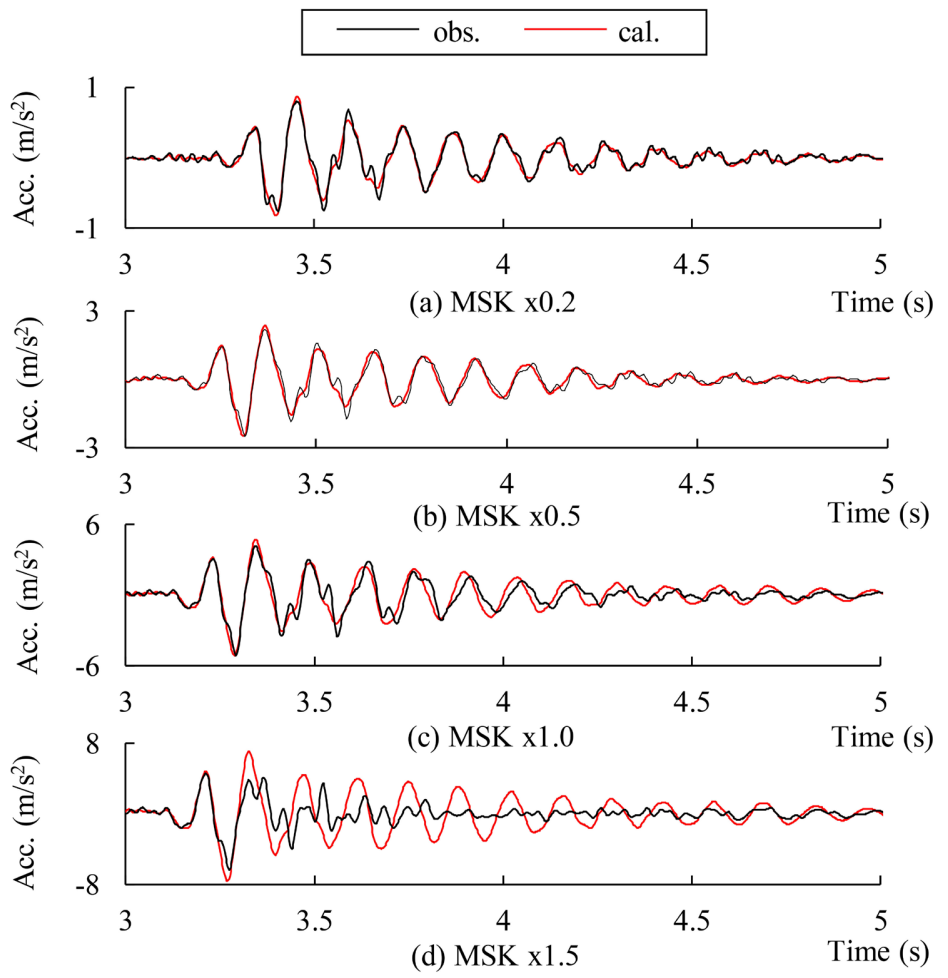

Figure 12. Acceleration history of mass calculated by model without separation of material surrounding the base side.

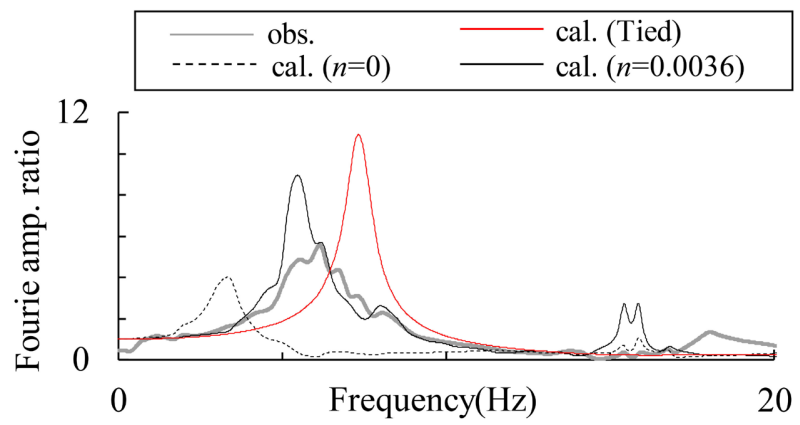

Figure 13. Fourier amplification factor of mass response to input motion (MSK x1.5). 


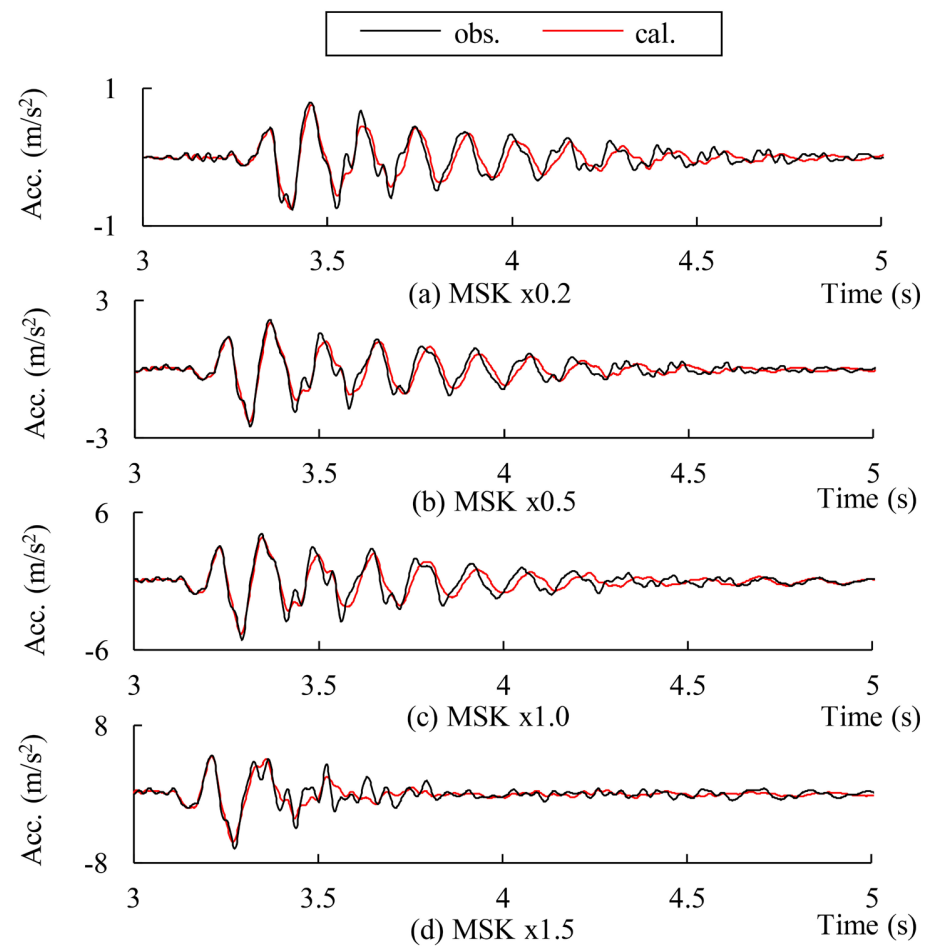

Figure 14. Acceleration history of mass calculated by model considering separation of material surrounding the base side.

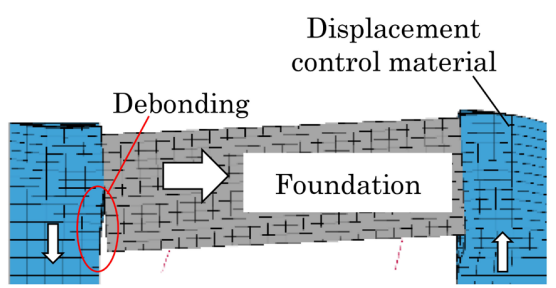

Figure 15. Deformation of material surrounding the base side.

Notably, the change in the distance between the magnetic poles due to the rocking of the base is only approximately $14 \%$ at the maximum, and the impact of modeling the repulsive force of the magnet as a linear spring on the analysis results is small.

\section{Seismic Response Analysis of Magnetically Levitated Full-Scale House Model}

In this section, we apply a seismic-isolated structure to a full-scale detached house model, and investigate its effectiveness according to seismic response analysis using the three-dimensional finite element method. In addition, we also compare analysis of the same building model using sliding-base isolation.

\subsection{Target Building Model}

The target building was a two-story full-scale detached house model. Figure 16 
shows the analytical model. It is a light gauge steel structure with a $2 \times 2$ span, approximately $8 \mathrm{~m}$ length on one side, and a $841.9 \mathrm{MN}$ superstructure in total weight. This is a two-mass shear model, and each layer was modeled with linear columns and structural elements with normal-trilinear hysteresis characteristics in parallel. The footing beams and footings were assumed to be rigid. The superstructure was subjected to mass-proportional damping with a damping constant of $1 \%$ with respect to the primary natural period. The input ground motion was the notification wave (random phase), which is the design ground motion that is equivalent to level 2 in Japan.

In this analysis, in addition to applying a seismic-isolated structure to the above building model, we compared a fixed base and the use of sliding-base isolation. Here, the sliding base had a perfect elasto-plastic hysteresis characteristic, and the yield load was assumed to be friction coefficient 0.08 [13].

\subsection{Various Factors of Magnet and Material Surrounding the Base Side}

The magnets levitating the house were assumed to have residual magnetic flux density of $1.2 \mathrm{~T}$, equivalent to a neodymium magnet, and a total of nine pairs, one pair under each footing, were installed to support the building. The shape of the magnets was a rectangle with a plane of $1 \mathrm{~m} \times 1 \mathrm{~m}$, and it was designed to be $0.3 \mathrm{~m}$ thick to levitate both the building and the magnet by $0.1 \mathrm{~m}$. Figure 17 shows the relationship between the repulsive force per pair of magnets and the distance between the magnetic poles. In the figure, the total weight of the building supported by each magnet and the magnets is shown by a dashed line as the repulsive force required of the magnets. In this analysis, the magnets are modeled as nonlinear elastic vertical springs, and we apply the force-deformation relationship obtained by approximating the repulsive force-distance between poles in Figure 17 in relationship to a polynomial equation.

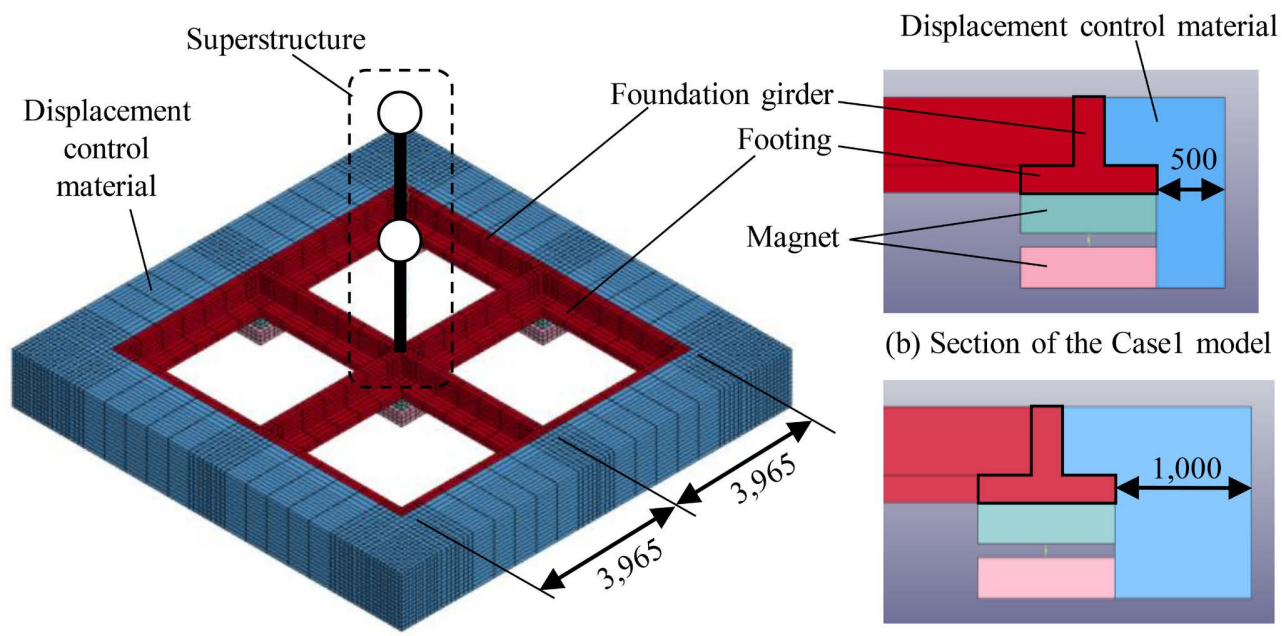

(a) Overview

(c) Section of the Case 2 model

Figure 16. 3D FE model of detached house. 


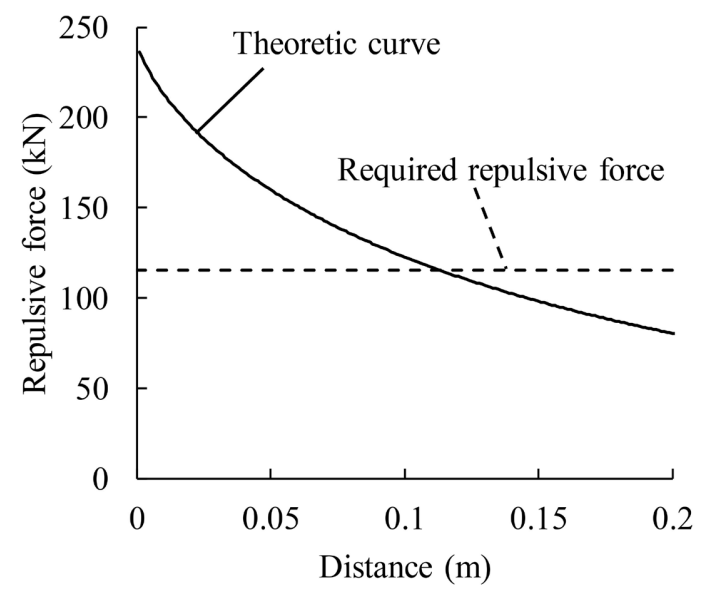

Figure 17. Relationship between magnetic force and distance of magnets.

The material surrounding the base side is a composite geomaterial made by mixing and polymerizing sand with magnesium acrylate [14]. Figure 18 shows the uniaxial compression test results for the composite geomaterial. This material is modeled as an elastic body because it has high linearity for large strains, similar to the material used in the experiment in Section 2. Figure 16(b) and Figure 16(c) show a cross-sectional view of members such as the footing beams and the material surrounding the base side. In the present analysis, two cases are analyzed: the narrow-width material surrounding the base side (Case 1: Figure 16(b)) and the wide-width one (Case 2: Figure 16(c)). Adhesion between the base and the material surrounding the base side is assumed. Stiffness proportional damping with a damping constant of $10 \%$ for the primary natural frequency is applied to the material surrounding the base side.

\subsection{Analysis Results}

\subsubsection{Comparison of Seismic-Isolated Model and Base-Fixed Model}

Figure 19 shows the acceleration time history waveform at the top of the superstructure in comparing Case 1 and Case 2 of the seismic-isolated model to the base-fixed model. The maximum acceleration of the seismic-isolated model is significantly reduced compared to the base-fixed model. Figure 20 shows the Fourier acceleration amplitude ratio of the response at the top of the superstructure to input in comparing the seismic-isolated model and the base-fixed model. Compared to the base-fixed model, the peak frequency of the Fourier acceleration amplitude ratio of the seismic-isolated model is low, and the peak value is smaller.

Figure 21 shows the maximum inter-story drift angle of the superstructure in comparing the seismic-isolated model and the base-fixed model. The inter-story drift angle of the seismic-isolated model is reduced compared to the base-fixed model. Case 2, in particular, in which the width of the material surrounding the base side is increased, shows a larger response-reducing effect than Case 1. 


\subsubsection{Comparison of Seismic-Isolated Model and Sliding-Base Isolation Model}

Figure 22 shows the acceleration time history waveform at the top of the superstructure in comparing Case 1 and Case 2 of the seismic-isolated model to the sliding-base isolation model. The maximum acceleration of the seismic-isolated model is reduced as much as in the sliding-base isolation model. Figure 23 shows the time waveform of the horizontal displacement of the building base. In the seismic-isolated model, the base returns to its original position on account of the restoring force of the material surrounding the base side, while residual displacement is observed in the sliding-base isolation model.

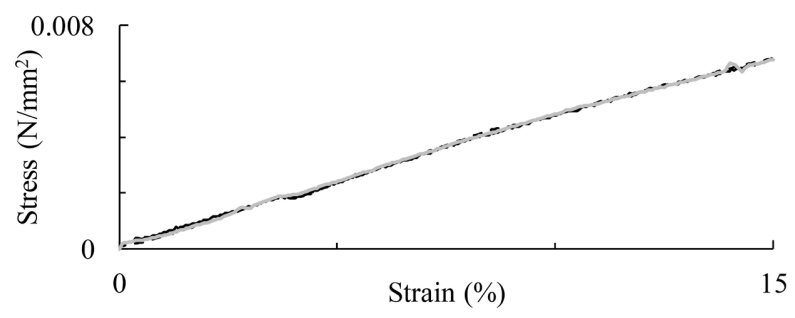

Figure 18. Result of uniaxial compression test for material surrounding the base side.

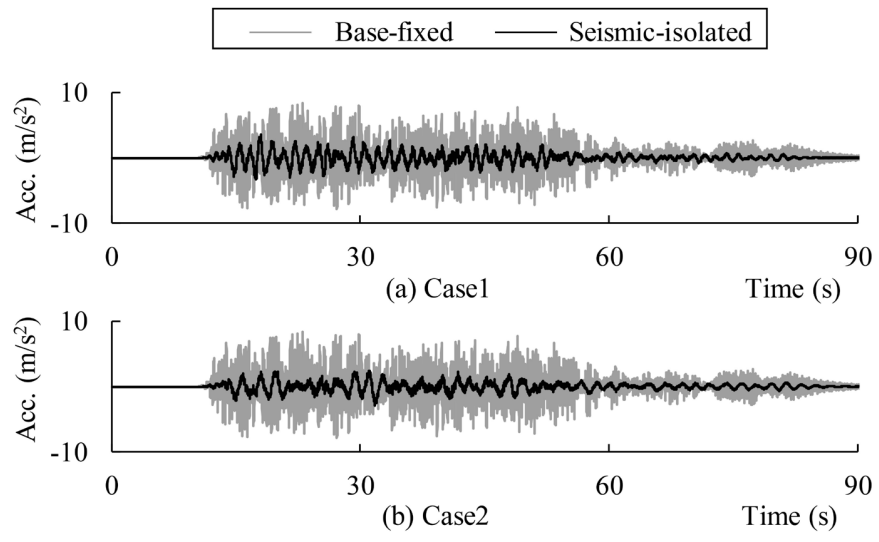

Figure 19. Comparison of acceleration history of RF for base-fixed and seismic-isolated models.

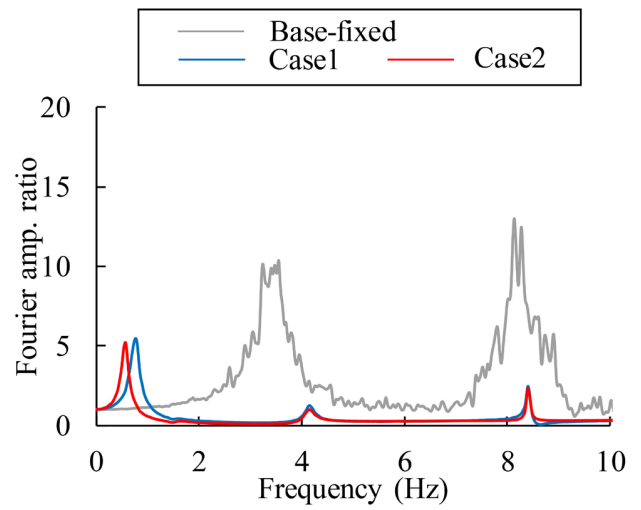

Figure 20. Fourier amplification factor of RF response to input motion for seismic-fixed and seismic-isolated models. 


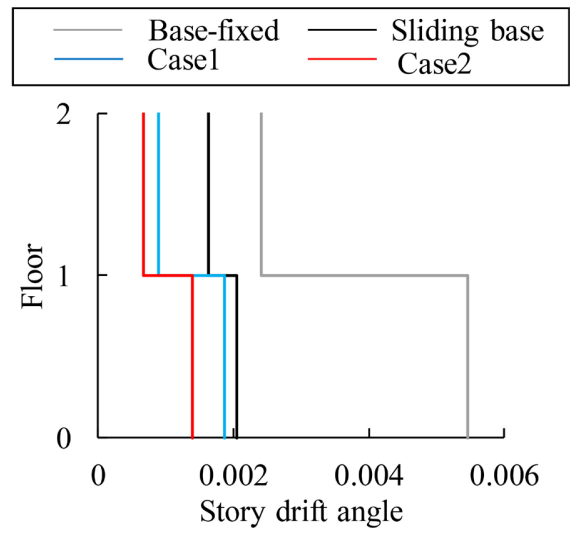

Figure 21. Maximum story drift angle of superstructure.

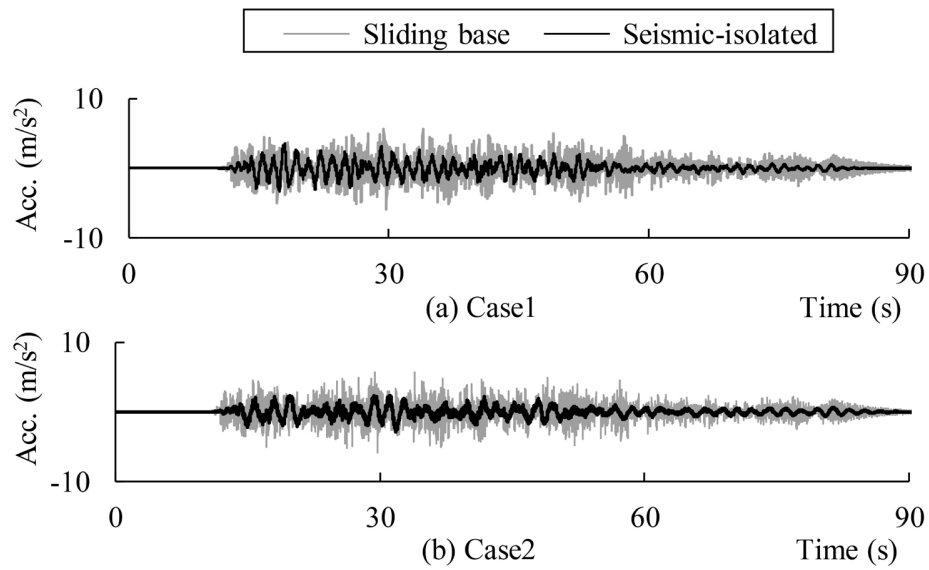

Figure 22. Comparison of acceleration history of RF for sliding base and seismic-isolated models.

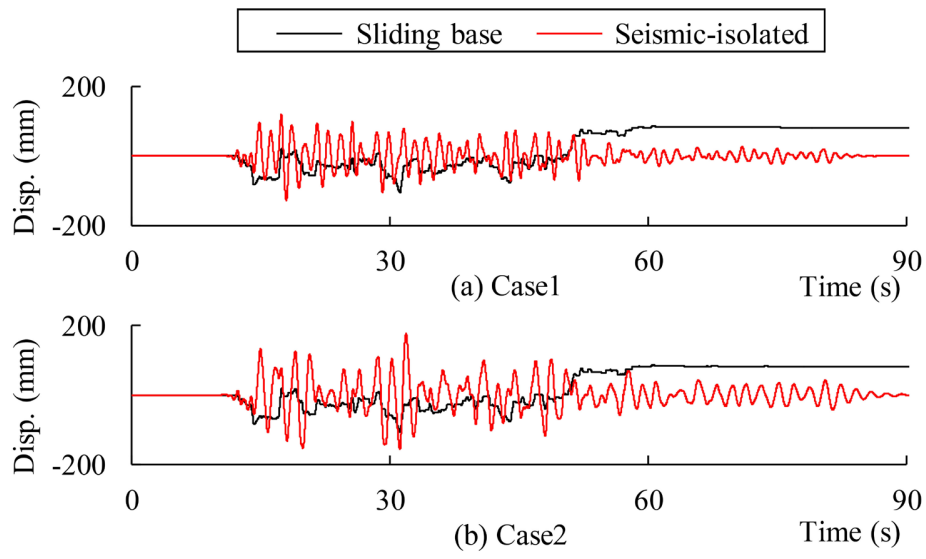

Figure 23. Comparison of displacement history of $1 \mathrm{~F}$ for sliding base and seismic-isolated models.

Figure 21 superimposes the maximum inter-story drift angle of the sliding-base isolation model. In the seismic-isolated model in Case 2, which enlarges the width of the material surrounding the base side, the inter-story drift angle is 
suppressed more than in the sliding-base isolation model.

From the above, it can be confirmed that the response-reducing effects of the seismic-isolated model are significant. In addition, it is believed that the suppression of residual displacement by the restoring force of the material surrounding the base side is superior in post-earthquake restoration.

\section{Conclusions}

In this study, a model shaking table experiment and three-dimensional finite element analysis were conducted to identify the effects of isolating a foundation base with magnetic levitation and reducing the seismic response of a detached house using the material surrounding the base side on the base sides. The obtained findings are shown below.

1) In the model shaking table experiment, the seismic-isolated model showed stable response, the superstructure was period-lengthened compared to the base-fixed model regardless of input amplitude. In addition, the amplitude of the acceleration response was significantly reduced.

2) It was shown that the characteristics of the repulsive force of a magnet of finite size can be evaluated by the dipole concept and the surface integral of Coulomb's law.

3) In the simulation of the experiment conducted using three-dimensional finite element analysis, it was possible to simulate the experimental results, including during large vibrations, by considering the contact condition between the base and the material surrounding the base side. In addition, it was found that the material surrounding the base side deforms and resists the movement of the building model three-dimensionally, which leads to a stabilized response in a seismic-isolated building.

4) The seismic response analysis of a full-scale detached house model showed that the response of the superstructure in the seismic-isolated model is reduced to the same or more as the sliding-base isolation model. Furthermore, it was shown that residual displacement is significantly suppressed by using the material surrounding the base side.

In further research, we intend to explore electromagnets controlled by electrical signals and building response during vertical motion input to enable application to a full-scale building.

\section{Acknowledgements}

In this study, we used the observation results of the National Research Institute for Earth Science and Disaster Resilience KiK-net Mashiki Station. In addition, we are sincerely grateful to Mr. Ryutaro Tsubouchi of Toagosei Co., Ltd. for his cooperation in the shaking table experiment.

\section{Conflicts of Interest}

The authors declare no conflicts of interest regarding the publication of this paper. 


\section{References}

[1] Research Society on Design Earthquake Ground Motion and Design Method of Building for Inland Earthquake Directly above Its Epicenter on Osaka (2015) Report of Research on Design Earthquake Ground Motion and Design Method of Building for Inland Earthquake Directly above Its Epicenter on Osaka Prefecture. Kansai Branch of Japan Structural Consultants Association, Osaka.

[2] Architectural Institute of Japan (1996) An Introduction to Dynamic Soil-Structure Interaction. Architectural Institute of Japan, Tokyo.

[3] Architectural Institute of Japan (2006) Seismic Response Analysis and Design of Buildings Considering Dynamic Soil-Structure Interaction. Architectural Institute of Japan, Tokyo.

[4] Kurosawa, E., et al. (2010) Mechalife People No. 25. Journal of the Society of Mechanical Engineers, 113, 451-455. https://doi.org/10.1299/jsmemag.113.1099 451

[5] Yasuda, M., Sato, E., Yamada, M., Kajiwara, K. and Hayatsu, M. (2017) Development of Three-Dimensional Seismic Isolation System Using Negative Stiffness Link Mechanism and Air Levitation Mechanism in Series (Overview of Prototype Experimental Device and Report on Full-Scale 3-D Seismic Excitation Experiment by E-Defense Shaking Table). Transactions of JSME, 83, 17-00057. https://doi.org/10.1299/jsmedmc.2017.235

[6] Katayama, K., et al. (1993) An Experimental Study on Dynamic Characteristics of Magnetic Floating Structure. Summaries of Technical Papers of Annual Meeting, Architectural Institute of Japan, Structure I, 619-620.

[7] Katamura, R., et al. (1995) Research on Electromagnetically-Levitated Vibration Isolation System (Part 6) Digital $\mathrm{H}^{\infty}$ Control of Three-Dimensional Vibration Isolation System. Summaries of Technical Papers of Annual Meeting, Architectural Institute of Japan, Structure II, 827-828.

[8] Tsuda, M., Kojima, T., Yagai, T. and Hamajima, T. (2007) Vibration Characteristics in Magnetic Levitation Type Seismic Isolation Device Composed of Multiple HTS Bulks and Permanent Magnets. IEEE Transactions on Applied Superconductivity, 17, 2059-2062. https://doi.org/10.1109/TASC.2007.901502

[9] Frame, T. and Pechey, A. (2012) A Magnetically Levitated Precise Pointing Mechanism for Application to Optical Communication Terminals. Optics and Photonics Journal, 2, 85-97. https://doi.org/10.4236/opj.2012.22012

[10] Miyamoto, Y., Shimamura, A., Fujii, S., Hoshizawa, F. and Kashiwa, H. (2018) A Fundamental Study on the Earthquake Response Reduction of Base-Isolated Foundation Backfilled Using an Improved Compound Geomaterial. Japan Architectural Review, 1, 56-66. https://doi.org/10.1002/2475-8876.1013

[11] Earnshaw, S. (1842) On the Nature of the Molecular Forces Which Regulate the Constitution of the Luminiferous Ether. Transaction of the Cambridge Philosophical Society, 7, 97-112.

[12] Sunagawa, S. (1997) Electromagnetics for Beginners. Baifukan, Tokyo.

[13] Hoshizawa, F., Fukaya, S., Miyamoto, Y., Nakata, S., Shimamura, A. and Miyake, T. (2014) Earthquake Response Reduction of a House with Sliding Bearing and Geo-Material Displacement Control System (Part2). Summaries of Technical Papers of Annual Meeting, Architectural Institute of Japan, Structure II, 525-526.

[14] Sato, A. and Miyamoto, Y. (2018) Study on Response Reduction of Cushioning on Retaining Wall in Collision of Base-Isolation Building. Summaries of Technical Papers of Annual Meeting, Architectural Institute of Japan, Structure II, 833-834. 